\title{
To Make the Teaching and Learning of Chinese Rhyme More Intuitive by Various Means
}

\author{
Xiao Jiugen ", Song Jie \\ Research Center of Language and Language Life, Jiangxi Normal University, Nanchang, China \\ Email address: \\ jxsdxjg666666@sina.com (Xiao Jiugen) \\ ${ }^{*}$ Corresponding author
}

To cite this article:

Xiao Jiugen, Song Jie. To Make the Teaching and Learning of Chinese Rhyme More Intuitive by Various Means. Science Innovation. Vol. 8, No. 5, 2020, pp. 155-160. doi: 10.11648/j.si.20200805.17

Received: October 28, 2020; Accepted: November 26, 2020; Published: November 27, 2020

\begin{abstract}
Chinese rhyme, a traditional basic subject, is a required course for graduate students majoring in Chinese language. Because of its ancient and complex content, it determines the particularity of its teaching and learning, so it is of great significance to study its teaching strategies and learning methods. Practice has proved that it is an important way to achieve good results in teaching and learning to adopt diversified methods with different contents. In its theory teaching and learning, the use of diagram, comparison, list, illustration and other methods, it can make the profound and complex content intuitive and popular. In its practice teaching and learning, the use of investigation, record, description, discussion and other methods, can make the theory into practical skills, so that learning to apply, the effect is very significant. Over the years, in the rhyme teaching and learning of graduate students, we have been in the first monarch road to seek work, aimed at trying to breakthrough the bottleneck explore a new road from theory to practice, and establish a paradigm, in order to realize the subject the ultimate goal of teaching and learning, and make it play its proper role in the new era.
\end{abstract}

Keywords: Chinese Rhyme, Postgraduate Teaching and Learning, Means of Diversification, Visualization, Results

\section{以多样化手段促使汉语音韵教学与学习直观化}

肖九根”, 宋洁

江西师范大学语言与语言生活研究中心, 南昌, 中国

邮箱

jxsdxjg666666@sina.com（肖九根）

摘要：汉语音韵这一传统的基础学科, 是高校汉语言专业研究生的必修课。因其内容的古奥性与复杂性, 这就决定了 其教学与学习的特殊性, 所以研究其教学策略与学习方法有着十分重要的意义。实践证明, 以其不同的内容采用多样 化的方法手段, 这是取得其教学与学习良好效果的重要途径。在其理论教学与学习中, 运用图解、比较、列表、例证 等方法, 能使古奥复杂的内容直观化、通俗化; 在其实践教学与学习中, 运用调查、记录、描写、探讨等方法, 能使 理论转化为实践技能, 这样学以致用, 成效十分显著。多年来, 在研究生的音韵教学与学习中, 我们一直从事开山辟 路的探求工作, 目的在于力图突破所面临的瓶颈, 从理论到实践探索出一条新路, 建立起一种范式, 以实现这一学科 教学与学习的终极目标, 使其在新时代发挥它应有的作用。

关键词: 汉语音韵, 教学与学习, 多样化手段, 直观化, 成效 


\section{1. 引言}

汉语音韵是一门传统的基础学科, 既是文科本科生的选 修课, 又是汉语言硕士生的必修课。由于这一学科不同于词 汇和语法, 有其独特性, 而这一独特性又决定了这一学科的 体系性与独立性。所以, 研究这一学科的教学策略与学习方 法, 对于提高其教学与学习效果有着十分重要的意义。

做任何事情都得讲究一定的方法。方法对头, 事情就会 朝着既定的成功方向发展; 方法不对, 不仅办不成事情, 有 时甚至还会南辕北辙, 永远无法取得成功。所以, 一个好的 科学方法既事半功倍, 又能取得很好的实际效果。毫无疑问, 汉语音韵的教学与学习也不得不讲究科学方法。实践证明, 以多样化的方法手段进行汉语音韵教学与学习, 努力使之直 观化, 这是这一学科取得良好效果的一条重要途径。

关于汉语音韵教学方法问题, 上世纪末以来, 有些学 者已经作过研究。诚然, 不可否认的是其中对音韵学习问 题也作了一定的探讨。例如, 刘廷武（1989）[1]、朱维德 （1992）[2]、肖永凤（1994）[3]、任晓肜（2011）[4]、 陈大为（2012）[5]、陈会兵（2013）[6]、胡佳佳（2018） [7]、周映映（2019）[8]和裴梦苏（2020）[9]等, 另两篇 论文如刘勇（2016）[10]和尹菲（2017）[11]则是针对基 础教育的。

纵观相关的研究成果, 有的是以汉语音韵作本体研究 的, 有的是以本科古汉语教学而研究的, 有的是以基础教 育阶段古诗文教学而研究的, 而以之作为研究生教学与学 习课改的独立学科进行研究的十分罕见, 亦可说尚属空白。 其中, 谈得较为深入的要算刘、朱、胡三位学者。刘、朱 的研究是以本科教学与学习为对象的, 具有一定的理论意 义和实践价值, 不过还未真正将它上升到一个独立学科体 系的理论高度和实际应用层面, 也不完全切合高层次 (如 研究生) 对象的实际; 胡的研究试图通过现代网络分析以 反观清·陈澧的系联法, 其研究上了一个较高的层次, 也 具方法论的意义, 但是不具汉语音韵这个学科的完整性、 系统性, 无法运用于汉语音韵教学与学习的全过程。为此, 我们的研究以研究生为对象, 对其教学与学习方法进行探 讨, 旨在实现高层人才运用这一学科以解决实际问题的终 极目标[12]。

\section{2. 音韵教学与学习最忌呆板的方法}

汉语音韵属于历史语音学, 从其产生、发展至今已有 1800 多年的历史。汉语音韵就其性质来说, 实属“口耳之 学”: 以声而听, 以听而思, 以思而悟, 而其听、其思、 其悟则是闻声之作由浅入深的三重境界[13]。因此，今天 人们学习研究汉语音韵存在诸多限制, 遇到很多困难在所 难免。诸如: 一是过去科学技术不发达, 古人说话的语音 无法录制保存下来, 这使今人无从知晓古人话语的实际音 值原貌; 二是汉语音韵是音韵学家根据历代韵文押韵、形 声字声符、古人对其时语音理论描述、外族古汉语借词资 料以及方音中的古音而构拟的, 实际上就是从汉字记录间 接地将古音韵的语音系统推求出来, 这使今人 (包括音韵 学家) 要准确地理解和掌握古音韵存在一定的困难; 三是
传统的汉语音韵古音的具体读法是以表意方块汉字表示 的, 不是拼音文字, 而要从方块汉字去寻求古代读音, 追 溯古音的发展变化, 这对今人而言也存在不少困难。因此, 要学习研究汉语音韵就不如当今汉语语音那样便捷。如今, 我们要学习研究现当代汉语语音可以直接听到或录制现 成而鲜活的语音材料, 这在古代是不可想象的。

就目前而言, 汉语言文学专业中仅有少数语言类教师 具备汉语音韵方面的素养, 甚至一些担任本科古汉语教学 的也不具有这方面的素质。据笔者每学期开课前调查表明, 语言学及应用语言学和文字学两个方向的硕士研究生, 每 届二十余人, 除极少数本科阶段对汉语音韵有所接触外, 大多为零基础, 即使汉语言文学专业的也不例外, 更何况 还有部分理工科或文科其它专业考入的。所以, 每当授课 之始, 教师总要设法扫除初学者的心理障碍, 提高其学好 汉语音韵的信心。

面对学习者背负的思想包补以及心理压力, 如何释放 出来, 这是授课前所要解决的一个极为重要的问题, 也是 教好、学好这一课程的前提条件。倘若不先行解决这一问 题, 之后的工作就会事倍功半, 得不偿失, 难以收到一个 好的教学与学习效果, 更谈不上运用所学的汉语音韵原理 去解决现实社会中的实际问题了。而要解决这一问题, 就 要激发初学者的学习热情与兴趣, 引发其求知欲, 提高其 自信心。为了解决这一难题, 老师要循循善诱, 竭力揭去 汉语音韵“绝学”的面纱, “对所授内容进行特殊处理, 力 求融知识性与趣味性于一体, 使之听而有味, 学而有 趣......将与之相关的趣闻轶事引进课堂教学。”[14]例如, 为了阐释汉语音韵合音现象与反切的区别, 教学中以三国 吕布为曹操擒获的故事为证来说明何为合音现象。吕布被 抓, 求刘备为之说情, 刘点头应允。当曹征询刘何以处置 时, 刘以吕数次叛主杀父示曹。曹决计杀之, 吕骂刘: “大 耳儿最匡信!”时吕愤怒，语速颇快，遂将“不可”合音成“匜” 了。再举古语将二声合为一字者不乏其例, 如常见的“何 不”为“盍”, “如是”为“尔”, “而已”为“耳”, “之乎”为“诸” 之类。又以汉语方言为例, 如北京人将“不用”谓之“甭”, 苏州人以“勿曾”说成“分”等典型的合音现象。汉语中的合 音现象, 仅是两个字音简单的拼装, 其介音乃至主要元音 常常不一致, 它与上字取其声、下字取韵调的反切法不能 等量齐观。这样, 在大家弄懂了合音现象的前提下, 老师 再讲反切原理, 就迎刃而解了。

由于课堂采用了寓理于趣事或例证的教学方法, 这不 仅解除了学习者的思想包䘞, 释放了大家的心理压力, 更 为重要的是引起了大家的极大兴趣, 使之感到汉语音韵不 但不神秘, 而且还是一个很有趣的学科。所以, “以这样 的方式教学, 即使再难的学科还会觉得它是“绝学”而害怕 吗，还会对它没有兴趣吗？显而易见, 答案是否定的。” 在教学实践中, 几乎每个知识点上均会设置一些趣闻轶事 或鲜活的方言例证材料, 深入浅出地讲解那些看似晦涩难 懂、实则一点即破的知识原理, 这“不仅打破了那种视汉 语音韵为“绝学”玄学”的怪论, 消除了学习者初始的畏难 心理, 激发了对这一学科的兴趣爱好, 而且还树立了学好 汉语音韵这一学科的信心, 从而为运用汉语音韵知识以解 决实际问题奠定了坚实的基础”[14]。 
诚然, 我们所说的趣闻轶事教学法, 仅是汉语音韵教 学中所采用的一种重要方法。这种方法对于学习者消除恐 惧心理, 激发学习兴趣, 树立战胜困难的信心十分有效, 但这并非是唯一的方法。

众所周知, 汉语音韵内容具有一定的古奥性和复杂性, 所以教学与学习方法也应多种多样, 而非简单地采用一种 方法就能有效地教好、学好这一学科。可以这样说, 汉语 音韵教学与学习最忌惮的是单一呆板的方法, 更忌板着脸 孔一味地讲解知识原理, 而应采取灵活多样的教学与学习 方法, 使之化难为易、鲜明直观, 这样才能收到好的预期 效果。

所以, 在阐述汉语音韵理论知识过程中, 为了将古奥 复杂的基本原理化为简单直观的通俗内容, 我们采用了图 解、比较、列表、例证等方法; 为了将所学的音韵理论知 识运用于解决实际问题, 我们又采取了考察、调查、记录、 描写、探讨等方法, 这样学以致用, 学练结合, 将所学理 论知识转化为实践技能, 就能很好地达到事半功倍的教学 与学习效果。

\section{3. 音韵教学与学习应以多样化手段使之直观化}

唯物辩证法认为, 任何事物都具有矛盾的两个方面 主要矛盾和次要矛盾, 只要抓住事物的主要矛盾, 事 物的次要矛盾就能迎刃而解。进行音韵教学与学习, 也要 抓主要矛盾的主要问题, 以相应的方法去各个击破。俗话 说一把钥匙开一把锁, 实际上说的就是这个道理。只有找 准了问题, 采取与之相适应的方法, 学习者才乐于接受, 从“外”入 “内”地深刻理解、领悟。所以, 在音韵教学与学 习中, 依据问题的性质特点采用不同的方法予以解决, 这 在方法论问题上具有十分重要的意义。

上已有述, 在音韵教学与学习中, 为了将其古奥复杂 的原理化为通俗直观的内容, 我们采用了图解、比较、列 表、例证、分析等方法进行阐述。

（1）图解法。图解法的优势在于直观性强, 一目了 然。在讲解“上字取其声, 下字取韵调”反切原理时, 为了 直观易懂, 我们就运用了这一方法。例如:

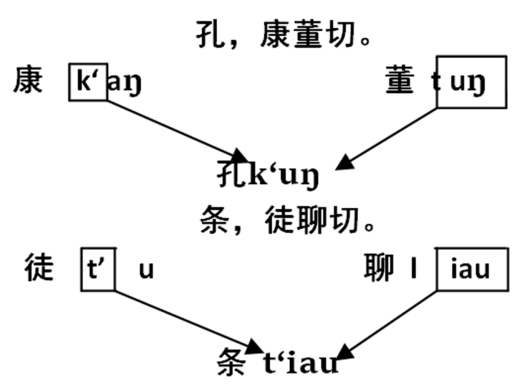

图1 反切法。

通过上述例子图解, 可以清楚地看到: 反切上字与被 切字为双声关系, 反切下字与被切字是叠韵同调关系。以 这种直观的方法图解, 无疑大大减少了反切原理理解的难 度, 使之变得通俗好懂。

清代学者陈澧以“系联法”考证古声类时云：“切语上 字同用者、互用者、递用者声必同类也。同用者, 如: ‘冬:
都宗切”, “当: 都郎切’, 同用“都”字也。互用者, 如: ‘当: 都郎切', 如: ‘都: 当孤切”, “都”当”二字互用也。递用 者, 如: ‘冬: 都宗切’, ‘都: 当孤切’, ‘冬”字用‘都”字, ‘都” 字用“当”字也。”若以此观之, 甚难理解; 如将之图解, 则 豁然开朗。示图:

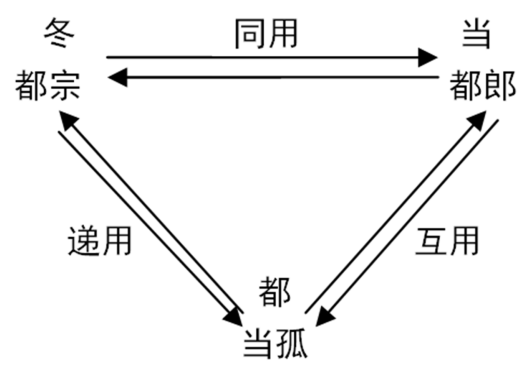

图2 系联法。

实际上, 声类的演变也是可以图解的, 如唇音的演变 过程:

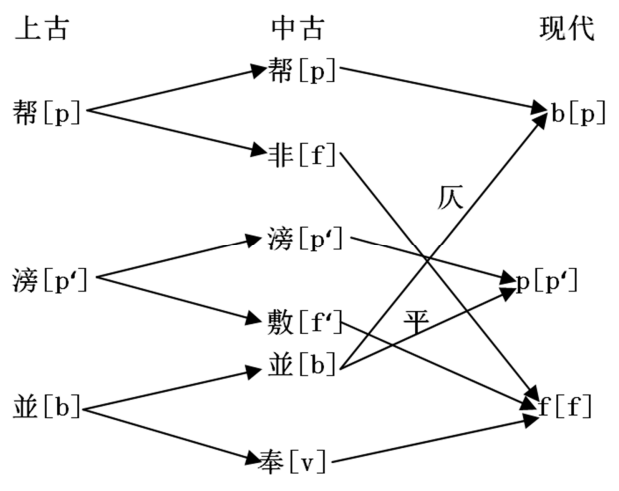

图3 唇音声母演变。

如此图解, 即使很长的历史发展过程, 很复杂的滟源 关系, 也一目了然。

（2）比较法。比较法的优势在于通过二者或多者的 对比, 确定其间是否具有对应关系, 其发展变化又是以一 个怎样的脉络进行, 这是常用的方法之一。例如, 汉语现 代音与中古音声韵调的比较, 就可以看出其发生的变化及 其存在的对应关系: 现代声类塞音 $\left[\mathrm{p} 、 \mathrm{p}^{\mathrm{c}}, \mathrm{t} 、 \mathrm{t}^{\mathrm{c}}, \mathrm{k}^{\mathrm{s}} \mathrm{k}^{\mathrm{c}}\right.$ ]、 塞擦音 [ts、ts' $\mathrm{ts}^{\prime} 、 \mathrm{ts}^{\prime}, \mathrm{t}$ 、 $、 \mathrm{t}^{\prime} \mathrm{c}^{\prime}$ 没有如中古音那样有清 $\left[p 、 p^{c}, t 、 t^{c}, k 、 k^{c}, t s 、 t s^{c}\right] 、$ 浊 $[b 、 d 、 g 、 d z]$ 的对立; 其韵类有阴声韵、阳声韵, 但不像中古音那样有入声韵[-p、 $-t 、-k]$, 等等。还可以将中古音与汉语方言进行对比, 从 而可直观地观察其相互关系, 总结其对应规律, 如: 粤方 言、客家方言与中古音在声韵调方面有着整齐的对应规律, 其韵类保留着完整的塞韵尾 [-p、-t、- $\mathrm{k}]$; 吴方言还留有古 浊塞音声类, 如“袍、抱、暴”等古音和吴语音皆为全浊“並” 母 $[\mathrm{b}] 、 “$ “徒、度、杜”等古音和吴语音读全浊“定”母 $[\mathrm{d}]$ 。比 较可见, 汉语方言 (尤其南方方言) 具有鲜明的存古特征。

(3) 列表法。列表的优势在于条理清晰, 分类明确, 直观而易于理解。在汉语音韵中, 古“等呼”与今“四呼”的 关系较为复杂, 仅以文字阐述不易理解, 如以表格的方式 则达到事半功倍的效果。

“等呼”演变为“四呼”如下表所示: 
表1 “等呼”与“四呼”的关系。

\begin{tabular}{clll}
\hline 今四呼 & 古一、二等 & 古三、四等 & 古两呼 \\
\hline 开 & a、an & ialia、ien & 开口呼 \\
齐 & & & \\
合 & ua、uan & yanlyan (iuan)、yen(iwen) & 合口呼 \\
撮 & &
\end{tabular}

又如, 《广韵》是承《切韵》而来的, 但二者的韵部 却有出入, 《广韵》多了十几个韵部。何以不一? 列表一 观，了然于心。
《切韵》与《广韵》韵部比较:

表2 《切韵》韵部与《广韵》韵部比较。

\begin{tabular}{|c|c|c|c|c|}
\hline 《切韵》 & 《广 & & & \\
\hline \multirow{2}{*}{ 真（谆）输（准）震（稕）质（术） } & 真 & 输 & 震 & 质 (开口) \\
\hline & 谆 & 准 & 稕 & 术 (合口) \\
\hline \multirow{2}{*}{ 寒（桓）旱（缓）翰（换）曷（末） } & 寒 & 旱 & 翰 & 曷（开口） \\
\hline & 桓 & 缓 & 换 & 末（合口） \\
\hline \multirow{2}{*}{ 歌 (戈) 哿（果）箇（过） } & 歌 & 哿 & 箇 & (开口) \\
\hline & 戈 & 果 & 过 & (合口) \\
\hline
\end{tabular}

观表则知, 二者的韵部之所以有异, 仅是《广韵》将其从开口呼中离析而出, 另立合口呼而已, 并非产生了新的韵部。

不仅如此, 实际上韵部如何演变为今音, 其过程皆能以表的形式展示, 如深摄各韵部演变为今音韵母（以平赅上 去），其演变条件如下：

表3 深摄韵部演变规律。

\begin{tabular}{|c|c|c|c|c|c|}
\hline \multirow{2}{*}{$\begin{array}{l}\text { 古音 } \\
\text { 摄 } \\
\end{array}$} & \multirow[b]{2}{*}{ 韵部 } & \multirow[b]{2}{*}{ 拟音 } & \multicolumn{3}{|l|}{ 今音 } \\
\hline & & & 音值 & 例字 & 与古声母关系 \\
\hline \multirow[b]{2}{*}{$\begin{array}{l}\text { 深 } \\
\text { 摄 }\end{array}$} & 侵 & Ǐĕm（开三） & $\begin{array}{l}\text { in } \\
\text { en } \\
\text { in }\end{array}$ & $\begin{array}{l}\text { 品林心今音 } \\
\text { 沉森深壬 } \\
\text { 禀 }\end{array}$ & $\begin{array}{l}\text { 帮组、泥组、精组、见系 } \\
\text { 知系 } \\
\text { 帮组个别字 }\end{array}$ \\
\hline & 缉 (入) & Ǐĕp（开三） & $\begin{array}{l}i \\
\gamma \\
i \\
u\end{array}$ & $\begin{array}{l}\text { 立习急邑 } \\
\text { 蛰涩 } \\
\text { 执汁湿十 } \\
\text { 入 }\end{array}$ & $\begin{array}{l}\text { 泥组、精组、见系 } \\
\text { 知系少数字 } \\
\text { 章组 } \\
\text { 日组少数字 }\end{array}$ \\
\hline
\end{tabular}

此外, 还有例证等方法。例如, 阐述《广韵》206韵 与今音韵母关系时, 我们例举了《吕氏春秋》中有关齐国 大臣东郭牙泄露军密的事例。齐桓公与管仲密谋伐莒之事, 东郭牙知晓后将之公布于众。其时莒国之“莒”为“鱼”[a]部, “口开而不阖”，读作开口呼[kia]; 而六朝以降，“莒”音逐 渐向高元化方向发展, 故今普音读作撮口呼“ju”, 此可谓 古今语音变化之殊。又如, 今普音“孩”[xai]读开口呼, “鞋”[6i 6 ]齐齿呼, 而方音二者皆为开口呼[xai]。为何呢? 此为方音存古性之故。《广韵》中, “孩”户来切（反切下 字“来”ai韵)，平咍，匣母；“鞋”户皆切（反切下字“皆” 亦ai韵），平皆，匣母，故二者古音基本相同，方音亦如 是。当然, 前文所说的趣闻轶事引证, 亦属此类。

至于分析法, 教学研究中阐述汉语音韵所具的性质特 点及其基本原理等皆离不开这一方法。实际上, 在上述的 各种方法中自始至终都贯穿了分析的方法。限于篇幅, 兹 不赘述了。

汉语音韵, 不仅内容古奥艰深, 理论知识性强, 而且 其间的关系也相互联系, 错综复杂。因此, 汉语音韵教学 与学习, 就应以特定的方法对应特定的内容。也就是说, 特定的钥匙只能打开特定的锁, 仅用一把钥匙是无法开启 所有锁的, 因为世上不存在真正的万能钥匙。所以, 唯有 方法得当, 才能将其内容处理得恰到好处, 使之通俗易懂, 学之有所得, 得之有所用。

\section{4. 音韵教学与学习应将理论知识同实际应用结 合起来}

任何一个学科的教学与学习都是为其实践服务的, 只有 将教与学同实践结合起来, 并以之解决实际问题, 才能达到 教与学的终极目标。因此, 汉语音韵理论知识的教学与学习, 仅是这个工作中的第一步。诚然, 这一步极为重要, 它为解 决实际问题奠定理论基础, 倘若没有这一工作, 要解决实际 问题就是纸上谈兵。但是, 对于汉语音韵而言, 仅有理论教 学与学习是远远不够的, 这难以实现其终极目标, 所以理论 教学与学习之后的实践教学与学习就显得尤为重要, 这是汉 语音韵从理想王国通往自由王国彼岸的必经之路。

如上所述, 在将汉语音韵理论知识运用于解决实际问 题过程中, 我们主要采用考察、调查、记录、描写、探讨 等实践的方法, 这样学以致用, 将所学理论知识转化为实 践的基本技能，多年来的实践证明其成效是十分显著的。

上述实践教学与学习方法, 实际上是一个以学生实践 为主体的系列连续过程。之所以说它是一个系列的连续过 程, 是因为那些实践工作不仅前后相续, 而且还环环相扣。 惟有这样, 才能将汉语音韵理论有机地融入于实践技能的 熟练掌握之中, 最终圆满地完成这一基本技能的实际工作。

为了完成这一基本技能的实践工作，我们是按以下方 法步骤进行的: 
（1）进行实地考察工作。汉语音韵理论教学与学习 完成之后, 每位学习者都要深入方言区的方言点（自选熟 悉的)有意识地观察人们是如何交流的, 并对其交流方式、 交际语音记录或录音, 当然这一切都是在对方毫无察觉的 情况下进行的, 这无需事先准备观察提纲什么的, 完全是 随机的, 这样做的主要目的是想捕捉其真实的语音面貌。 多年来, 每届学习者都是如此, 现在还一直在做, 而且一 定要坚持下去。

(2) 进行田野调查与记录收集工作。从理论学习到 实践活动, 从课堂授课到田野调查, 这是实现理论知识转 化为实践技能的一个重要途径, 也是我们从事汉语音韵教 学研究的重要方法之一, 它对于提高学习者分析问题、解 决实际问题的能力有着不可替代的作用, 是教学研究具有 原创性与实用性的重要保证。所以, 方言田野调查、记录 方音与收集语料工作是其中的重要环节, 如果没有这一环 节, 后面的工作就无法进行下去, 也难以取得实际成效。 而要进行田野调查、方音记录与语料收集工作, 先要确定 方言调查点, 有的放矢地制定一个切实的调查纲要, 找准 发音地道纯正的合作者, 并作好记音或录音的准备工作。 当然, 不少调查者以中国社科院语言所《方言调查字表》 为蓝本调查方言声韵调及其例字的, 也有人在国家语委语 保工程调查材料的基础上根据实情勘酌增减进行调查的。

(3) 进行方音整理与描写工作。将调查所收集的方 音材料整理、归纳, 使之条理化, 以便从中找出规律, 进 而对其音系进行系统描写。反映方音的基本面貌, 既要真 实地描写方音声韵调的构成, 又要抓住方音的基本特点, 更为重要的还要揭示方音发展变化的基本规律, 从而得出 经得起实践检验的基本结论。其中, 描写方言音系是一项 基础性工作。有了这个基础, 我们不仅可以对其进行共时 方面的分析研究, 诸如考察方言变调、编集同音字汇、分 辨文白异读、洞察方音细微等, 还能进一步地对其作历时 变化的考源工作, 如将其与上古或中古音作比照分析, 探 其源流关系等。就一般情况而言, 如果一个学习者能全力 做好这一工作，那他不仅具备了汉语音韵的基本素养，而 且还有较强的实践科研能力了。

在实际工作中, 还有一个方面不容忽视, 那就是对存 在的问题进行探讨, 这是汉语音韵教学中教师从实际出发, 以问题引导学习者深入思考的一个重要环节, 它将学习者 引入一个相互切磋、共同探讨的更高阶段和理想境界。每 届一、二十位研究生, 在实际的调查研究中总会遇到这样 或那样的问题, 这些问题有的凭个人的能力能够解决, 有 的则无法解决。每当调查研究结束之后, 我们就集中在一 起, 将各类问题提到桌面上共同讨论。在问题的探讨过程 中, 大家畅所欲言, 各抒己见, 时而唇枪舌箭, 时而你驳 我辩, 互不相让, 颇受启发, 许多问题就是在这样的集思 广益、激烈争论中得到解决的。

\section{5. 结语}

概而言之, 针对汉语音韵学科的独特性, 我们阐述了 如何根据不同内容运用各种不同的教学策略与学习方法 使之直观化、通俗化问题, 目的在于力图打破音韵教学与
学习长期以来所面临的瓶颈, 从理论到实践探索出一条新 路, 建立起一种范式。

俗话说得好, 他山之石, 可以攻玉。也就是说, 研究 生的音韵教学与学习所采用的不应仅仅局限于本学科常 用的那几种方法, 而应广泛地借鉴或尝试运用其它学科 (包括理工科) 的不同方法, 只要行之有用, 能把深奥难 懂的汉语音韵理论知识简易化、直观化, 让学习者听之有 趣, 学之好懂, 用之有效, 能熟练掌握并运用其原理解决 实际问题, 为其从事教学与研究打下坚实基础, 这就达到 了汉语音韵教学与学习的终极目标。

近些年来, 我们所从事的就是这样一项开山辟路的探 求工作, 这不仅揭开了“绝学”的面纱, 还在研究方言音韵 方面取得了可喜的成绩。据不完全统计, 至目前为止, 研 究生以方言音韵作学位论文的达 20 多篇, 另有近 10 篇方言 音韵学术论文发表在国内外核心刊物上, 这在教者无人教、 学者害怕学的过去是不可想象的, 也是前所未有的一个奇 迹。

学习研究汉语音韵, 不仅仅因为它是一个传统的基础 学科, 更为重要的它还是历史传承的一种民族文化。为了 彰显民族文化自信与文化向心力, 我们需要学习研究它, 这对于重塑文化自信、促进中华文化传播具有重要的现实 意义[15]。我们深信, 汉语音韵这一古老的传统文化, 在 新时代定会绽放出新的活力, 为培养社会主义建设人才发 挥它应有的作用。

\section{致谢}

基金项目: 江西省学位与研究生教育教学改革研究项 目“揭开“绝学”面纱, 继承传统文化一一关于研究生课程 《音韵学》教改问题的几点思考”阶段性成果（编号: JXYJG-2014-038）。

\section{参考文献}

[1] 刘廷武. 音韵学教学初探[J].四川师范学院学报(哲社科 版)1989(03)。

[2] 朱维德. 音韵教学杂谈[J].古汉语研究1992(03)。

[3] 肖永风.今古音对照以简驭繁——古汉语音韵教学方法初 探[J].六盘水师范高等专科学校学报1994(02)。

[4] 任晓䑣.高等师范院校古代汉语音韵教学探索 [J].内蒙古师 范大学学报(教科版)2011(09)。

[5] 陈大为.高校古代汉语课程音韵学教学内容与方法改革初 探[J].吉林教育学院学报2012(06)。

[6] 陈会兵.《古代汉语》课程“音韵”部分教学方案探索 [J].重庆 三峡学院学报2013(06)。

[7] 胡佳佳.网络分析方法在音韵学教学中的应用——以广韵》 反切系联为例 $[J]$.励耘语言学刊2018(02)。 
[8] 周映映. 谈从音韵切入古典诗词教学 [J]. 语文教学之友 2019(03)。

[9] 裴梦苏.走出“绝学”困境——当下通行古代汉语教材中音韵 学内容编写策略研究 $[J]$. 长春师范大学学报2020(01)。

[10] 刘勇.高中语文古诗文教学中音韵学知识作用举隅 [J].课程 教育研究2016(23)。

[11] yin fei. Research on teaching the classical chinese poems in junior hign school_ based on the perspective of phonology [D]. Master's thesis of Zhejiang Normal University. 2017.
[12] Xiao Jiugen, Xie Lu. Combine Common Language with Dialect, Promote the Popularization of Chinese Phonology [J]. Science Innovation, 2020 (08).

[13] 傅修延,邱宗珍.因声而听、因听而思和因听而悟——试论闻 声之作的三重境界 [J]. 江西师范大学学报 (哲社科 版)2019(02)。

[14] Jiugen Xiao, Guangyu Yu. Revealing "Lost Knowledge" of Chinese Phonology through Anecdotes [J]. Science Innovation. Science Publishing Group, USA. 2017.

[15] 曹顺庆,王昌宇.文化自信与中国文论建设[J].江西师范大学 学报(哲社科版) 2018(06)。 